\title{
Astrophysical and theoretical physics implications from multimessenger neutron star observations
}

\author{
Hector O. Silva, ${ }^{1,2}$ A. Miguel Holgado, ${ }^{3,4,5}$ Alejandro Cárdenas-Avendaño, ${ }^{2,6}$ and Nicolás Yunes ${ }^{2}$ \\ ${ }^{1}$ Max-Planck-Institut für Gravitationsphysik (Albert-Einstein-Institut), Am Mühlenberg 1, D-14476 Potsdam, Germany \\ ${ }^{2}$ Department of Physics and Illinois Center for Advanced Studies of the Universe, \\ University of Illinois at Urbana-Champaign, Urbana, Illinois 61801, USA \\ ${ }^{3}$ Department of Physics and McWilliams Center for Cosmology, \\ Carnegie Mellon University, Pittsburgh, Pennsylvania, 15213, USA \\ ${ }^{4}$ Department of Astronomy, University of Illinois at Urbana-Champaign, Urbana, Illinois 61801, USA \\ ${ }^{5}$ National Center for Supercomputing Applications, University of Illinois at Urbana-Champaign, Urbana, Illinois 61801, USA \\ ${ }^{6}$ Programa de Matemática, Fundación Universitaria Konrad Lorenz, 110231 Bogotá, Colombia
}

\begin{abstract}
The Neutron Star Interior Composition Explorer (NICER) recently measured the mass and equatorial radius of the isolated neutron star PSR J0030+0451. We use these measurements to infer the moment of inertia, the quadrupole moment, and the surface eccentricity of an isolated neutron star for the first time, using relations between these quantities that are insensitive to the unknown equation of state of supranuclear matter. We also use these results to forecast the moment of inertia of neutron star $A$ in the double pulsar binary J0737-3039, a quantity anticipated to be directly measured in the coming decade with radio observations. Combining this information with the measurement of the tidal Love number with LIGO/Virgo observations, we propose and implement the first theory-agnostic and equation-of-state-insensitive test of general relativity. Specializing these constraints to a particular modified theory, we find that consistency with general relativity places the most stringent constraint on gravitational parity violation to date, surpassing all other previously reported bounds by 7 orders of magnitude and opens the path for a future test of general relativity with multimessenger neutron star observations.
\end{abstract}

Introduction.- Neutron stars are some of the most extreme objects in nature. Their mass (typically around $1.4 \mathrm{M}_{\odot}$ ) combined with their small radius (between $10-14 \mathrm{~km}$ ) result in interior densities that can exceed nuclear saturation density $\left(\rho \geqslant 2.8 \times 10^{14} \mathrm{~g} / \mathrm{cm}^{3}\right)$, above which exotic states of matter can arise [1]. Neutron stars are, next to black holes, the strongest gravitational field sources known, with typical gravitational potentials that are 5 orders of magnitude larger than that of the Sun. These properties make neutron stars outstanding laboratories to study both matter and gravity in situations out of reach in terrestrial and Solar System experiments.

Our current poor understanding of the supranuclear equation of state translates, via the equations of stellar equilibrium, to a large variability on observable properties of neutron stars, such as their masses and radii [2]. This variability increases if one lifts the assumption that Einstein's theory of general relativity is valid in the strong-gravity environment of neutron star interiors [3]. Modifications to general relativity generically predict new equations of stellar equilibrium, which, when combined with uncertainties on the nuclear equation of state, jeopardize attempts to test Einstein's theory with isolated, neutron star observations.

One possibility to circumvent this issue is to explore whether relations between neutron-star observables that are insensitive to either (or both) the equation of state and the gravitational theory exist. Fortunately, they do. For instance, when properly nondimensionalized, the moment of inertia $(I)$, the rotational quadrupole moment $(Q)$ and the tidal Love number $(\lambda)$ of neutron stars show a remarkable degree of equationof-state insensitivity, at a level below $1 \%[4,5]$. These "ILove-Q" relations also exist in some modified theories of gravity, although they are different from their general relativity counterparts [6].

We here combine the first measurements [7, 8] by
NICER [9] of both the mass $(M)$ and equatorial radius $\left(R_{\mathrm{e}}\right)$ of the isolated pulsar PSR J0030+0451 $[10,11]$ with known equation-of-state insensitive relations involving the compactness $\mathscr{C}=G M /\left(R_{\mathrm{e}} c^{2}\right) \quad$ (see, for instance Refs. [12-15]) to infer a number of astrophysical and theoretical physics consequences. Before doing so, let us explain how these relations are obtained.

Quasiuniversal relations. - Neutron stars can have short rotation periods of the order of milliseconds, so their surfaces are oblate instead of spherical. The inclusion of this effect is of critical importance to accurately model the thermal xray waveform that NICER observes, since the $\mathrm{x}$-rays are produced by hotspots at the star's surface $[16,17]$. The canonical approach to model relativistic rotating stars was developed in the 1970s [18, 19]. In this approach, the star's rotation is treated as a small perturbation $\varepsilon=f / f_{0} \ll 1$, involving the star's rotation frequency $f$ and its characteristic mass-shedding frequency $f_{0}=\left(G M / R_{\mathrm{e}}^{3}\right)^{1 / 2} /(2 \pi)$. Rotating stars are then found by perturbing in $\varepsilon$ an otherwise nonrotating star, which can be obtained by solving the TolmanOppenheimer-Volkoff equations [20]. This slow-rotation approximation is well justified for most neutron stars with astrophysically relevant spins. Even for a prototypical millisecond pulsar with $f=700 \mathrm{~Hz}, M=1.4 \mathrm{M}_{\odot}$ and $R_{\mathrm{e}}=11 \mathrm{~km}$, one has $\varepsilon=0.37$. In the case of PSR J0030+0451, its rotation frequency is known to be $f_{\star}=205.53 \mathrm{~Hz}[10,11]$, so $\varepsilon_{\star}=0.14$, when one uses the best-fit $M$ and $R_{\mathrm{e}}$ values obtained by NICER [7, 8]. Henceforth, a " $\star$ " indicates observables associated with PSR J0030+0451 .

Using this technique, we numerically calculated over a thousand neutron star solutions to order $\varepsilon^{2}$ in this perturbative scheme, using a broad set of 46 different equations of state [21, 22], as detailed in the Supplemental Material (SM). From these solutions, we then numerically computed the mo- 
ment of inertia $I$, the rotational quadrupole moment $Q$, the surface eccentricity $e$, and the electric-type, $\ell=2$, tidal Love number $\lambda$, which is the dominant parameter in the description of tidal effects in the late inspiral of neutron star binaries [23-25]. We nondimensionalized these quantities through division by the appropriate factors of $M$ and dimensionless spin $\chi=\left(2 \pi f_{0}\right) G \bar{I} M / c^{3}$, namely: $\bar{I}=c^{4} I /\left(G^{2} M^{3}\right)$, $\bar{Q}=-c^{4} Q /\left(G^{2} M^{3} \chi^{2}\right)$ and $\bar{\lambda}=c^{10} \lambda /(G M)^{5}$. The surface eccentricity $e$ is dimensionless by definition, given in terms of the star's equatorial $R_{\mathrm{e}}$ and polar $R_{\mathrm{p}}$ radii as $e=\left[\left(R_{\mathrm{e}} / R_{\mathrm{p}}\right)^{2}-\right.$ $1]^{1 / 2}$ [14]. The relations between these nondimensional quantities are strongly insensitive to the equation of state. Because of the small value of $\varepsilon_{\star}$ we can neglect higher order in spin corrections in this expression.

The first step in using the approximately universal relations on NICER's first observation is to derive equation-of-stateinsensitive relations between the observables $\{\bar{I}, \bar{Q}, \bar{\lambda}, e\}$, with respect to the compactness $\mathscr{C}$. Details of these " $\mathscr{C}$ relations" are given in the SM. Our plan of attack is then clear: use the publicly available Markov Chain Monte Carlo (MCMC) $M-R_{\mathrm{e}}$ samples $[26,27]$ for the best-fit model inferred by two independent analysis [7, 8] of the NICER data [28]. Although each group modeled the surface hotspots differently and used different sampling methods, their results are consistent with each other. Here we use the results for the three-hotspot model inferred by Miller et al. [8] and the favored single temperature, two-hotpot ST+PST model from Riley et al. [7] to obtain a posterior distribution for the compactness, and then use the approximately universal relations to infer other astrophysical quantities. We detail this procedure next.

Astrophysical implications. - We begin by constructing a posterior distribution $P(\mathscr{C} \mid$ NICER $)$ for the compactness $\mathscr{C}$ of PSR J0030+0451, using the MCMC chains [26, 27]. With this posterior in hand, we then use the $\mathscr{C}$ relations to inferred posterior distributions for $\{\bar{I}, \bar{\lambda}, \bar{Q}, e\}$.

The implementation of such an inference procedure requires a particular scheme, and we here follow a proposal that accounts for the approximately universal nature of the relations [22]. In this scheme, the maximum relative error of each fitting function defines the half width of the $90 \%$ credible interval of a Gaussian distribution centered at each fitted value. The posterior distribution for each dimensionless quantity is then calculated using the corresponding $\mathscr{C}$ relation and the posterior distribution of the compactness, after marginalizing over the latter. From these posteriors and using the same procedure described above, we can also construct posteriors for the dimensionful versions of these quantities by a change of variables, marginalize over the nuisance variables mass $M$ and radius $R_{\mathrm{e}}$, and then do a final rescaling of the posterior by $\varepsilon$ $(=0.14)$ for the surface eccentricity $e$ and by $\varepsilon^{2}$ for the rotational quadrupole moment $Q$. We refer to the SM for details.

The resulting mean and $1 \sigma$ intervals of these parameters (both the nondimensionalized and the dimensionful versions) are shown in Table I; see the SM section for plots of the inferred posteriors. The reported confidence intervals in all of these quantities account for both the approximate nature of the universal relations and the uncertainties in NICER's observation. These results are the first inferences on the moment

\begin{tabular}{lrc}
\hline \hline Parameter & \multicolumn{2}{c}{ Miller et al. } \\
\hline $\bar{I}_{\star}(10)$ & $1.31_{-0.11}^{+0.13}$ & $1.42_{-0.19}^{+0.26}$ \\
$\bar{\lambda}_{\star}\left(10^{2}\right)$ & $4.97_{-1.28}^{+1.92}$ & $6.75_{-2.69}^{+5.52}$ \\
$\bar{Q}_{\star}$ & $5.92_{-0.61}^{+0.73}$ & $6.50_{-1.03}^{+1.38}$ \\
\hline$I_{\star}\left(10^{45} \mathrm{~g} \mathrm{~cm}^{2}\right)$ & $1.71_{-0.48}^{+0.64}$ & $1.42_{-0.53}^{+0.81}$ \\
$Q_{\star}\left(10^{43} \mathrm{~g} \mathrm{~cm}^{2}\right)$ & $1.49_{-0.45}^{+0.63}$ & $1.27_{-0.49}^{+0.74}$ \\
$e_{\star}\left(10^{-1}\right)$ & $1.56_{-0.21}^{+0.25}$ & $1.58_{-0.28}^{+0.29}$ \\
\hline \hline
\end{tabular}

TABLE I. Inferred properties of PSR J0030+0451 using equationof-state-insensitive relations combined with the MCMC samples by Miller et al. [26] and Riley et al. [27]. We report the values within 1 standard deviation from the mean, representing approximately $68 \%$ confidence intervals. These values are the first inferences of the moment of inertia, the eccentricity, the Love number, and the quadrupole moment of an isolated neutron star.

of inertia, the surface eccentricity, the Love number and the quadrupole moment of an isolated neutron star.

We can also use NICER's observation combined with the I-C relation to estimate the moment of inertia of PSR J07373039A $\left(I_{1.3381}\right)$, where the subscript refers to this pulsar's measured mass of $M=(1.3381 \pm 0.0007) \mathrm{M}_{\odot}$ [29]. The double pulsar J0737-3039 is expected to provide the first direct neutron star measurement of the moment of inertia [30]. This system is the only double-pulsar observed to date, which makes it an unique laboratory for binary stellar astrophysics [31, 32]. Moreover, an accurate measurement of $I_{1.3381}$ in combination with its known mass is expected to strongly constrain the nuclear equation of state around once and twice nuclear saturation density [33].

To predict the moment of inertia of PSR J0737-3039A from NICER's observation of PSR J0030+0451, we first need to obtain an estimate for the compactness $\mathscr{C}_{1.3381}$ of PSR J0737-3039A. This can be approximated by the substitution $\left\{M, R_{\mathrm{e}}\right\} \mapsto\left\{M_{0}=1.3381 \mathrm{M}_{\odot}, R_{\mathrm{e}}\right\}$ at each MCMC sample [26] and then computing $\mathscr{C}_{M_{0}}$. This yields an approximation to the distribution of compactness for a system with mass $M_{0}$, which is assumed known and identical to PSR J0030+0451. This procedure is only justified as long as $M_{0}$ is very close to $M_{\star}$, as in the case of PSR J07373039A, whose inferred mass $\left(M_{0}=1.3381_{-0.0007}^{+0.0007} \mathrm{M}_{\odot}\right)$ [29] is within the $1 \sigma$ credible interval of both NICER's mass inference $\left(M_{\star}=1.34_{-0.16}^{+0.15} \mathrm{M}_{\odot}[7]\right.$ and $\left.M_{\star}=1.44_{-0.14}^{+0.15} \mathrm{M}_{\odot}[8]\right)$.

With an estimate of the compactness of PSR J0737-3039A, we can now obtain a prediction for PSR J0030+0451's moment of inertia repeating the procedure applied to PSR J0030+0451. Figure 1 shows our result using both

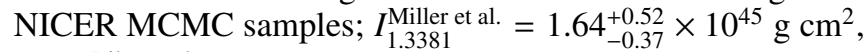
and $I_{1.3381}^{\text {Riley et al. }}=1.68_{-0.48}^{+0.53} \times 10^{45} \mathrm{~g} \mathrm{~cm}^{2}$, together with two other independent predictions [34, 35]. All predictions are consistent with one another. The anticipated future independent measurement of $I_{1.3381}$ from continued radio timing of PSR J0737-3039A will provide another test for nuclear theory and enable an "I-Love test" of gravity, the latter of which we define next.

Theoretical physics implications. - The combination of the inference of $I$ with NICER data described above, and the in- 


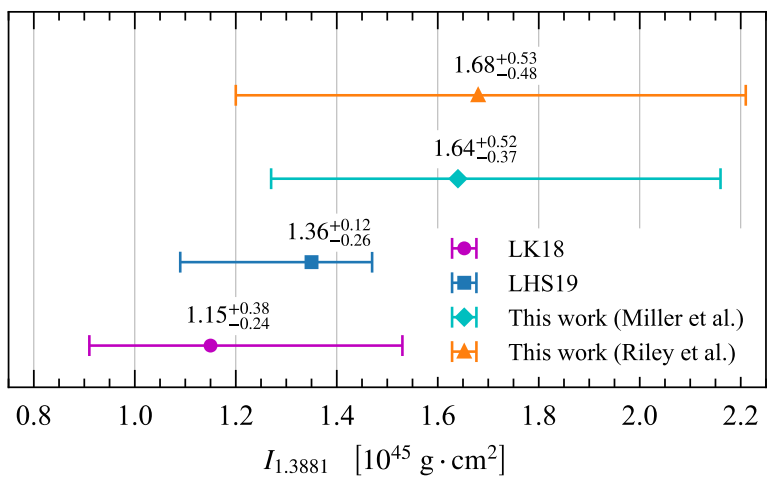

FIG. 1. Predictions for the moment of inertia of PSR J0737-3039A. We compare our predicted $I_{1.3381}$ using both the MCMC samples from Miller et al. [26] and Riley et al. [27] against: (i) Landry and Kumar [34] ("LK18"), which used binary Love [38] and I-Love relations with the tidal-deformability constraints from binary neutronstar merger GW170817 [36], and (ii) Lim et al. [35] ("LHS19") which carried out Bayesian modeling of a number of equations of state. The larger moment of inertia that we predict is due to the larger radii favored by an $M \approx 1.4 \mathrm{M}_{\odot}$ neutron star by NICER's observation relative what is inferred by the two other methods, as $I \sim M R_{\mathrm{e}}^{2}$.

dependent measurement of $\lambda[36]$ by the LIGO/Virgo Collaboration from the binary neutron-star merger GW170817 [37], allows for the first implementation of an I-Love test [4]. This test would be the first multimessenger test of general relativity with neutron star observables.

The idea of an I-Love test is as follows [4, 5] (see Fig. 2). Consider two independent inferences of $\bar{I}_{1.4}$ and $\bar{\lambda}_{1.4}$ for a $1.4 \mathrm{M}_{\odot}$ neutron star. In the $(\bar{I}, \bar{\lambda})$ plane, these measurements yield a $90 \%$ confidence error box. If the I-Love relation in general relativity, including its small equation-of-state variability, does not pass through this error box, then there is evidence for a violation of Einstein's theory, regardless of the underlying equation of state. Moreover, if any theory of gravity predicts an I-Love curve that also does not pass through this error box for a given value of its coupling constants, then the I-Love test places a constraint on the couplings of this theory, which is also independent of the equation of state.

Such a test, however, requires the inference of the tidal deformability and the moment of inertia of a neutron star of the same mass. The LIGO/Virgo Collaboration used gravitational wave data to infer the tidal deformability of a $1.4 \mathrm{M}_{\odot}$ neutron star to be $\bar{\lambda}_{1.4}=190_{-120}^{+390}$ at $90 \%$ confidence [39], obtained under the assumptions that the binary components were described by the same equation of state and were slowly spinning. We can use NICER's data to infer the moment of inertia of a $1.4 \mathrm{M}_{\odot}$ neutron star with the same techniques we used to predict the moment of inertia of PSR J0737-3039A. For concreteness, we use the results from Miller et al. [8, 26], but we verified (see the SM for detail) that our conclusions are essentially the same had we used the results from Riley et al. [7, 27]. We find that $\mathscr{C}_{1.4}=0.159_{-0.022}^{+0.025}$ and $\bar{I}_{1.4}=14.6_{-3.3}^{+4.5}$ at $90 \%$ confidence. An important underlying assumption behind both inferences is that general relativity is the correct theory of gravity. The rationale behind this test is detailed in the SM.

Since carrying out such a test on a theory-by-theory basis would, in general, be complicated and time consuming, we here develop and implement a useful parametrization of the I-Love test. From Newtonian gravity, we know that $\bar{I}$ scales with $\mathscr{C}^{-2}$, whereas $\bar{\lambda}$ scales with $\mathscr{C}^{-5}$. Therefore, $\bar{I}=C_{\bar{I}} \bar{\lambda}^{2 / 5}$, with $C_{\bar{I} \bar{\lambda}} \approx 0.52$ a constant that depends on the equation of state very weakly [5]. This calculation can be extended, systematically, in a post-Minkowskian expansion, i.e., an expansion in powers of $\mathscr{C} \ll 1$ [40]. The outcome is that both $\bar{I}$ and $\bar{\lambda}$ can be written as a power series in $\mathscr{C}$ and then be combined (as just described in the Newtonian limit) to obtain $\bar{I}=\bar{I}(\bar{\lambda})$. The resulting I-Love relation has the same degree of equationof-state independence as the original I-Love relation [4]. For our neutron star catalog, a parametrization in general relativity of the form

$$
\bar{I}_{\mathrm{GR}}=\bar{\lambda}^{2 / 5}\left(c_{0}+c_{1} \bar{\lambda}^{-1 / 5}+c_{2} \bar{\lambda}^{-2 / 5}\right),
$$

with $c_{0}=0.584, c_{1}=0.980, c_{2}=2.695$, is sufficient to reproduce our numerical data with mean relative error $\left\langle\epsilon^{\bar{I}}\right\rangle \leqslant 2 \times 10^{-3}$. The prefactor $\bar{\lambda}^{2 / 5}$ is the Newtonian result, while the powers of $\bar{\lambda}^{-1 / 5}$ inside parenthesis are relativistic (post-Minkowskian) corrections because $\bar{\lambda}^{-1 / 5} \propto \mathscr{C} \lesssim 0.2$. Given this, we then propose a minimal deformation of the Einsteinian parametrization in Eq. (1) of the form

$$
\bar{I}_{\mathrm{p}}=\bar{I}_{\mathrm{GR}}+\beta \bar{\lambda}^{-b / 5}, \quad \beta \in \mathbb{R}_{+}, \quad b \in \mathbb{Z},
$$

where $\beta$ and $b$ are deformation parameters that control the magnitude and type of the deviations from general relativity in the I-Love relation respectively. Such a parametrization is similar to that successfully used in gravitational-wave tests of general relativity by the LIGO/Virgo Collaboration, the parametrized post-Einsteinian framework [41].

We performed such a test of general relativity through the procedure described earlier. First, we see that the I-Love relation in general relativity does indeed pass this null-test and it is consistent with the error box. Second, we considered $b \in[-2,5]$, where the lower limit is set by requiring no deviations at the Newtonian level and the upper limit is set for simplicity. We then fixed $b$ and calculated what the corresponding value of $\beta=\beta_{\text {crit }}$ is, above which the parametrized I-Love relation (2) would be in tension with the inferred $\left(\bar{I}_{1.4}, \bar{\lambda}_{1.4}\right)$ region at $90 \%$ confidence. Our results are summarized in Fig. 2, where the numbers in parenthesis correspond to $\left(b, \beta_{\text {crit }}\right)$. We stress that our results for $b \leqslant 0$ are of course dependent on the posterior used for $\bar{\lambda}_{1.4}$. If one treated the tidal deformabilities as independent free parameters in the waveform model [37], then the $\bar{\lambda}_{1.4}$ posterior would not have a lower limit, allowing all curves with $b \leqslant 0$ to be consistent with both observations.

With these theory-agnostic constraints in hand, we can now map them to specific theories and place constraints on their coupling parameters. As an example, let us consider dynamical Chern-Simons gravity, a theory that modifies general relativity by introducing gravitational parity violation [42]. This theory has found applications to several open problems in cosmology, such as the matter-antimatter asymmetry and 


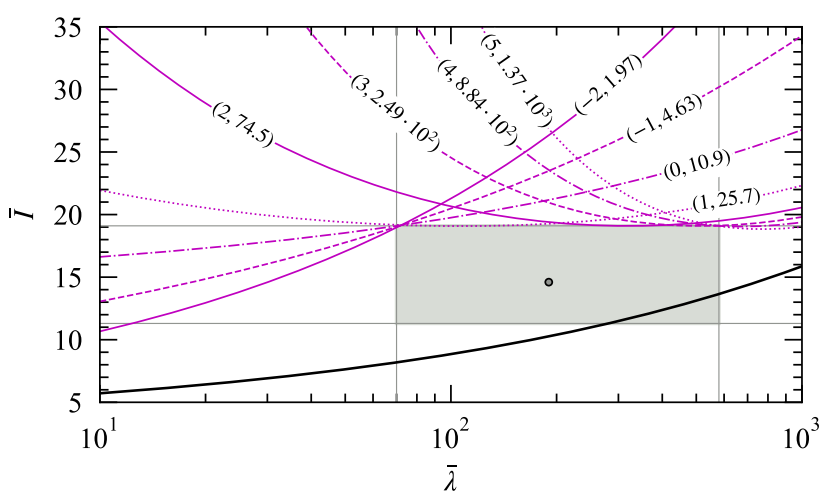

FIG. 2. Multimessenger test of general relativity using the parametrized I-Love relation. The vertical (horizontal) lines delimit the $90 \%$ confidence region (shaded) for $\bar{\lambda}_{1.4}$ [39] $\left(\bar{I}_{1.4}\right.$, this work), while the circle marks the median $(190,14.6)$. The solid black line corresponds to the I-Love relation in general relativity [Eq. (1)] and is consistent with the inferred values of $\bar{I}_{1.4}, \bar{\lambda}_{1.4}$ at $90 \%$ confidence. Starting from $b=-2$ and moving clockwise, we show the parametrized I-Love curves $\left(b, \beta_{\text {crit }}\right)$, where $b \in[-2,5]$ and $\beta_{\text {crit }}$ is the critical value of $\beta$ above which the parametrized I-Love relation [Eq. (2)] fails to pass by the $90 \%$ confidence region in the plane. Here we used the value of $\bar{I}_{1.4}$ inferred using the results by Miller et al. $[8,26]$. We found similar results using the results by Riley et al. [7, 27] (See SM).

leptogenesis [43-46]. It also arises in several approaches to quantum gravity, such as string theory [47] and loop quantum gravity [48-50]. Mathematical well-posedness requires the theory to be treated as an effective field theory [51]. In this formalism, one works in a small-coupling approximation $\zeta \equiv 16 \pi \alpha^{2} \mathscr{R}^{-4} \ll 1$, where $\mathscr{R}=\left[c^{2} R_{\mathrm{e}}^{3} /(G M)\right]^{1 / 2}$ is the curvature length scale associated with a neutron star (in our case), and where $\alpha$ is a coupling constant with units of length squared, such that $\zeta$ is dimensionless. This theory modifies Einstein's only when gravity is strong, and thus, it passes all Solar System constraints, being only extremely weakly constrained by Gravity Probe B and the LAGEOS satellites, and table-top experiments, to $\alpha^{1 / 2} \leqslant 10^{8} \mathrm{~km}$ [52-54]. This theory has also evaded gravitational-wave tests [55], making it a key target to test the constraining power of our new I-Love test.

Let us now map the theory-agnostic deformation of the ILove relations in Eq. (2) to dynamical Chern-Simons gravity, though this methodology could be applied to other theories as well. As we discuss in the SM, the I-Love relation in this theory can be described by Eq. (2) with $b_{\mathrm{cs}}=4$ and $\beta_{\mathrm{cS}}=$ $6.15 \times 10^{-2} \bar{\xi}$, where $\bar{\xi}=16 \pi \alpha^{2} / M^{4}$. We can now use our theory-agnostic constraints on $\beta$ to place a constraint on $\alpha$, the coupling constant of dynamical Chern-Simons gravity. Using the constraint on $\beta$ when $b=4$, namely $\beta_{\text {crit }} \leqslant 8.84 \times 10^{2}$, and applying the mapping, yields $\beta_{\mathrm{CS}}=6.15 \times 10^{-2} \bar{\xi} \leqslant 8.84 \times 10^{2}$, or simply

$$
\alpha^{1 / 2} \leqslant 8.5 \mathrm{~km}
$$

at $90 \%$ credibility, if the theory is to be consistent with the observational bounds on $\bar{I}_{1.4}$ and $\bar{\lambda}_{1.4}$. Using the mean value $\mathscr{C}_{1.4}=0.159$, which implies the mean equatorial radius $R_{1.4}=$ $13.0 \mathrm{~km}$, we also find that $\zeta \leq 0.23$ when using Eq. (3), implying that the small-coupling approximation is indeed satisfied. This bound is 7 orders of magnitude stronger than any previous constraints and it is unlikely to be improved upon with foreseeable gravitational-wave observations [56].

Conclusions and outlook.- NICER's observation of PSR J0030+0451 allows the extraction of new astrophysical and theoretical physics inferences when one uses equation-ofstate-insensitive relations. We have here shown the first inferences of the moment of inertia, the quadrupole moment, the surface eccentricity and the Love number of an isolated neutron star. We have also been able to perform the first theoryagnostic and equation-of-state independent test of general relativity by combining NICER and LIGO/Virgo's observations. This test, in turn, was leveraged to produce the most stringent constraint on gravitational parity violation, improving previous bounds by 7 orders of magnitude. This robust methodology can be applied to future multimessenger observations of neutron stars with NICER and gravitational wave observatories, with important implications to nuclear astrophysics and theoretical physics.

Acknowledgments. We thank Toral Gupta, Fred Lamb, Philippe Landry, and Helvi Witek for various discussions. We also thank Cole Miller, Sharon Morsink and Kent Yagi for suggestions that improved this work. We thank the NICER Collaboration for making [26, 27] publicly available. H.O.S, A.C.-A. and N.Y. are supported by NASA Grants Nos. NNX16AB98G, 80NSSC17M0041, 80NSSC18K1352 and NSF Award No. 1759615. A.C.-A. also acknowledges funding from the Fundación Universitaria Konrad Lorenz (Project 5INV1). A.M.H. was supported by the DOE NNSA Stewardship Science Graduate Fellowship under Grant No. DE-NA0003864.
[1] G. Baym, T. Hatsuda, T. Kojo, P. D. Powell, Y. Song, and T. Takatsuka, "From hadrons to quarks in neutron stars: a review," Rept. Prog. Phys. 81, 056902 (2018).

[2] F. Özel and P. Freire, "Masses, Radii, and the Equation of State of Neutron Stars," Ann. Rev. Astron. Astrophys. 54, 401-440 (2016).

[3] E. Berti et al., "Testing General Relativity with Present and Future Astrophysical Observations," Class. Quant. Grav. 32,
243001 (2015).

[4] K. Yagi and N. Yunes, "I-Love-Q," Science 341, 365-368 (2013).

[5] K. Yagi and N. Yunes, "I-Love-Q Relations in Neutron Stars and their Applications to Astrophysics, Gravitational Waves and Fundamental Physics," Phys. Rev. D 88, 023009 (2013).

[6] D. D. Doneva and G. Pappas, "Universal Relations and Alternative Gravity Theories,” Astrophys. Space Sci. Libr. 457, 737- 
806 (2018).

[7] T. E. Riley et al., "A NICER View of PSR J0030+0451: Millisecond Pulsar Parameter Estimation,” Astrophys. J. Lett. 887, L21 (2019).

[8] M. Miller et al., "PSR J0030+0451 Mass and Radius from NICER Data and Implications for the Properties of Neutron Star Matter," Astrophys. J. Lett. 887, L24 (2019).

[9] K. C. Gendreau et al., "The Neutron star Interior Composition Explorer (NICER): design and development," in Proc. SPIE, Society of Photo-Optical Instrumentation Engineers (SPIE) Conference Series, Vol. 9905 (2016) p. 99051H.

[10] A. N. Lommen, A. Zepka, D. C. Backer, M. McLaughlin, J. C. Cordes, Z. Arzoumanian, and K. Xilouris, "New pulsars from an Arecibo drift scan search,” Astrophys. J. 545, 1007 (2000).

[11] Z. Arzoumanian et al. (NANOGrav), "The NANOGrav 11-year Data Set: High-precision timing of 45 Millisecond Pulsars," Astrophys. J. Suppl. 235, 37 (2018).

[12] J. M. Lattimer and M. Prakash, "The Equation of State of Hot, Dense Matter and Neutron Stars," Phys. Rept. 621, 127-164 (2016), arXiv:1512.07820 [astro-ph.SR].

[13] A. Maselli, V. Cardoso, V. Ferrari, L. Gualtieri, and P. Pani, "Equation-of-state-independent relations in neutron stars," Phys. Rev. D 88, 023007 (2013).

[14] M. Bauböck, E. Berti, D. Psaltis, and F. Özel, "Relations Between Neutron-Star Parameters in the Hartle-Thorne Approximation," Astrophys. J. 777, 68 (2013).

[15] C. Breu and L. Rezzolla, "Maximum mass, moment of inertia and compactness of relativistic stars," Mon. Not. Roy. Astron. Soc. 459, 646-656 (2016).

[16] S. M. Morsink, D. A. Leahy, C. Cadeau, and J. Braga, "The Oblate Schwarzschild Approximation for Light Curves of Rapidly Rotating Neutron Stars," Astrophys. J. 663, 1244-1251 (2007).

[17] S. Bogdanov et al., "Constraining the Neutron Star MassRadius Relation and Dense Matter Equation of State with NICER. II. Emission from Hot Spots on a Rapidly Rotating Neutron Star,” Astrophys. J. 887, L26 (2019).

[18] J. B. Hartle, "Slowly rotating relativistic stars. 1. Equations of structure," Astrophys. J. 150, 1005-1029 (1967).

[19] J. B. Hartle and K. S. Thorne, "Slowly Rotating Relativistic Stars. II. Models for Neutron Stars and Supermassive Stars," Astrophys. J. 153, 807 (1968).

[20] S. L. Shapiro and S. A. Teukolsky, Black holes, white dwarfs, and neutron stars: The physics of compact objects (1983).

[21] J. S. Read, B. D. Lackey, B. J. Owen, and J. L. Friedman, "Constraints on a phenomenologically parametrized neutronstar equation of state," Phys. Rev. D 79, 124032 (2009).

[22] B. Kumar and P. Landry, "Inferring neutron star properties from GW170817 with universal relations," Phys. Rev. D 99, 123026 (2019).

[23] T. Mora and C. M. Will, "Numerically generated quasiequilibrium orbits of black holes: Circular or eccentric?" Phys. Rev. D 66, 101501 (2002).

[24] E. E. Flanagan and T. Hinderer, "Constraining neutron star tidal Love numbers with gravitational wave detectors," Phys. Rev. D 77, 021502 (2008).

[25] T. Hinderer, "Tidal Love numbers of neutron stars," Astrophys. J. 677, 1216-1220 (2008).

[26] M. C. Miller et al., "NICER PSR J0030+0451 IllinoisMaryland MCMC Samples," (2019).

[27] T. E. Riley, A. L. Watts, S. Bogdanov, P. S. Ray, R. M. Ludlam, S. Guillot, Z. Arzoumanian, C. L. Baker, A. V. Bilous, D. Chakrabarty, K. C. Gendreau, A. K. Harding, W. C. G. Ho, J. M. Lattimer, S. M. Morsink, and T. E. Strohmayer, "A
NICER View of PSR J0030+0451: Nested Samples for Millisecond Pulsar Parameter Estimation," (2020).

[28] S. Bogdanov et al., "Constraining the Neutron Star MassRadius Relation and Dense Matter Equation of State with NICER. I. The Millisecond Pulsar X-Ray Data Set,” Astrophys. J. Lett. 887, L25 (2019).

[29] M. Kramer et al., "Tests of general relativity from timing the double pulsar," Science 314, 97-102 (2006), arXiv:astroph/0609417 [astro-ph].

[30] M. Kramer and N. Wex, "The double pulsar system: A unique laboratory for gravity,” Class. Quant. Grav. 26, 073001 (2009).

[31] I. H. Stairs, S. E. Thorsett, R. J. Dewey, M. Kramer, and C. A. McPhee, "The Formation of the Double Pulsar PSR J07373039A/B,” Mon. Not. Roy. Astron. Soc. 373, L50-L54 (2006).

[32] R. D. Ferdman et al., "The double pulsar: evidence for neutron star formation without an iron core-collapse supernova," Astrophys. J. 767, 85 (2013).

[33] J. M. Lattimer and B. F. Schutz, "Constraining the equation of state with moment of inertia measurements," Astrophys. J. 629, 979-984 (2005).

[34] P. Landry and B. Kumar, "Constraints on the moment of inertia of PSR J0737-3039A from GW170817,”' Astrophys. J. 868, L22 (2018).

[35] Y. Lim, J. W. Holt, and R. J. Stahulak, "Predicting the moment of inertia of pulsar J0737-3039A from Bayesian modeling of the nuclear equation of state," Phys. Rev. C 100, 035802 (2019).

[36] B. P. Abbott et al. (LIGO Scientific, Virgo), "Properties of the binary neutron star merger GW170817," Phys. Rev. X 9, 011001 (2019).

[37] B. P. Abbott et al. (LIGO Scientific, Virgo), "GW170817: Observation of Gravitational Waves from a Binary Neutron Star Inspiral," Phys. Rev. Lett. 119, 161101 (2017).

[38] K. Yagi and N. Yunes, "Binary Love Relations," Class. Quant. Grav. 33, 13LT01 (2016).

[39] B. P. Abbott et al. (LIGO Scientific, Virgo), "GW170817: Measurements of neutron star radii and equation of state," Phys. Rev. Lett. 121, 161101 (2018).

[40] T. K. Chan, A. P. O. Chan, and P. T. Leung, "I-Love relations for incompressible stars and realistic stars," Phys. Rev. D 91, 044017 (2015).

[41] N. Yunes and F. Pretorius, "Fundamental Theoretical Bias in Gravitational Wave Astrophysics and the Parametrized PostEinsteinian Framework,” Phys. Rev. D 80, 122003 (2009).

[42] R. Jackiw and S. Y. Pi, "Chern-Simons modification of general relativity," Phys. Rev. D 68, 104012 (2003).

[43] S. Weinberg, “A Tree Theorem for Inflation,” Phys. Rev. D 78, 063534 (2008).

[44] J. Garcia-Bellido, M. Garcia-Perez, and A. Gonzalez-Arroyo, "Chern-Simons production during preheating in hybrid inflation models," Phys. Rev. D 69, 023504 (2004).

[45] S. H. Alexander and S. J. Gates Jr., "Can the string scale be related to the cosmic baryon asymmetry?" JCAP 06, 018 (2006).

[46] S. H.-S. Alexander, M. E. Peskin, and M. M. Sheikh-Jabbari, "Leptogenesis from gravity waves in models of inflation," Phys. Rev. Lett. 96, 081301 (2006).

[47] M. Adak and T. Dereli, "String-Inspired Chern-Simons Modified Gravity In 4-Dimensions,” Eur. Phys. J. C 72, 1979 (2012).

[48] A. Ashtekar, A. Balachandran, and S. Jo, "The CP Problem in Quantum Gravity,” Int. J. Mod. Phys. A 4, 1493 (1989).

[49] S. Mercuri and V. Taveras, "Interaction of the Barbero-Immirzi Field with Matter and Pseudo-Scalar Perturbations," Phys. Rev. D 80, 104007 (2009), arXiv:0903.4407 [gr-qc].

[50] L. Smolin and C. Soo, "The Chern-Simons invariant as the natural time variable for classical and quantum cosmology," Nucl. 
Phys. B 449, 289-316 (1995), arXiv:gr-qc/9405015.

[51] T. Delsate, D. Hilditch, and H. Witek, "Initial value formulation of dynamical Chern-Simons gravity," Phys. Rev. D 91, 024027 (2015).

[52] S. Alexander and N. Yunes, "Chern-Simons Modified General Relativity,” Phys. Rept. 480, 1-55 (2009).

[53] K. Yagi, N. Yunes, and T. Tanaka, "Slowly Rotating Black Holes in Dynamical Chern-Simons Gravity: Deformation Quadratic in the Spin,” Phys. Rev. D 86, 044037 (2012), [Erratum: Phys. Rev.D89,049902(2014)].

[54] Y. Nakamura, D. Kikuchi, K. Yamada, H. Asada, and N. Yunes, "Weakly-gravitating objects in dynamical Chern-Simons gravity and constraints with Gravity Probe B," Class. Quant. Grav. 36, 105006 (2019).

[55] R. Nair, S. Perkins, H. O. Silva, and N. Yunes, "Fundamental Physics Implications for Higher-Curvature Theories from Binary Black Hole Signals in the LIGO-Virgo Catalog GWTC-1," Phys. Rev. Lett. 123, 191101 (2019).

[56] S. H. Alexander and N. Yunes, "Gravitational wave probes of parity violation in compact binary coalescences," Phys. Rev. D 97, 064033 (2018).

[57] H. T. Cromartie et al., "Relativistic Shapiro delay measurements of an extremely massive millisecond pulsar," Nat. Astron. 4, 72-76 (2019).

[58] M. C. Miller, C. Chirenti, and F. K. Lamb, "Constraining the Equation of State of High-density Cold Matter Using Nuclear and Astronomical Measurements," Astrophys. J. 888, 12 (2020).

[59] K. Yagi, L. C. Stein, N. Yunes, and T. Tanaka, "Isolated and Binary Neutron Stars in Dynamical Chern-Simons Gravity,” Phys. Rev. D 87, 084058 (2013), [Erratum: Phys. Rev.D93,no.8,089909(2016)].

[60] K. Yagi and N. Yunes, "Approximate Universal Relations for Neutron Stars and Quark Stars,” Phys. Rept. 681, 1-72 (2017).

[61] Y. Ali-Haïmoud and Y. Chen, "Slowly-rotating stars and black holes in dynamical Chern-Simons gravity,” Phys. Rev. D 84, 124033 (2011).

[62] T. Gupta, B. Majumder, K. Yagi, and N. Yunes, "I-Love-Q Relations for Neutron Stars in dynamical Chern Simons Gravity," Class. Quant. Grav. 35, 025009 (2018).

[63] T. Damour and J. H. Taylor, "Strong field tests of relativistic gravity and binary pulsars," Phys. Rev. D 45, 1840-1868 (1992).

[64] T. Damour and G. Esposito-Farèse, "Tensor multiscalar theories of gravitation,” Class. Quant. Grav. 9, 2093-2176 (1992).

[65] T. Damour and G. Esposito-Farèse, "Nonperturbative strong field effects in tensor - scalar theories of gravitation," Phys. Rev. Lett. 70, 2220-2223 (1993).

[66] T. Damour and G. Esposito-Farèse, "Tensor - scalar gravity and binary pulsar experiments," Phys. Rev. D 54, 1474-1491 (1996), arXiv:gr-qc/9602056.

[67] D. Anderson, P. Freire, and N. Yunes, "Binary pulsar constraints on massless scalar-tensor theories using Bayesian statistics," Class. Quant. Grav. 36, 225009 (2019), arXiv:1901.00938 [gr-qc].

[68] P. Wagle, N. Yunes, D. Garfinkle, and L. Bieri, "Hair loss in parity violating gravity,” Class. Quant. Grav. 36, 115004 (2019), arXiv:1812.05646 [gr-qc].

[69] N. Yunes, D. Psaltis, F. Özel, and A. Loeb, "Constraining Parity Violation in Gravity with Measurements of NeutronStar Moments of Inertia,” Phys. Rev. D 81, 064020 (2010), arXiv:0912.2736 [gr-qc].

[70] H. Sotani, "Pulse profiles from a pulsar in scalar-tensor gravity,” Phys. Rev. D 96, 104010 (2017), arXiv:1710.10596 [astro-
ph.HE].

[71] H. O. Silva and N. Yunes, "Neutron star pulse profiles in scalartensor theories of gravity," Phys. Rev. D 99, 044034 (2019), arXiv:1808.04391 [gr-qc].

[72] R. Xu, Y. Gao, and L. Shao, "Strong-field effects in massive scalar-tensor gravity for slowly spinning neutron stars and application to X-ray pulsar pulse profiles,” Phys. Rev. D 102, 064057 (2020), arXiv:2007.10080 [gr-qc].

[73] H. O. Silva and N. Yunes, "Neutron star pulse profile observations as extreme gravity probes," Class. Quant. Grav. 36, 17LT01 (2019), arXiv:1902.10269 [gr-qc]. 


\section{- Supplemental Material -}

Equation of state catalog.- We consider the large catalog of nuclear equations of state of [22], supplemented by the non-duplicates from [21]. To this set of 85 equations of state, we impose the following observational consistency criteria. First, the equation of state must be consistent with the $90 \%$ confidence region of the two-dimensional marginalized posterior $\left(M, R_{\mathrm{e}}\right)$ reported by the LIGO/Virgo collaboration [39] for each component of the binary neutronstar merger GW170817. Second, the equation of state must be consistent with the $90 \%$ confidence region of the two-dimensional marginalized posterior $\left(M, R_{\mathrm{e}}\right)$ reported by NICER [7, 8] for PSR J0030+0451. Third, the equation of state must allow for neutron stars with masses above $M_{\max } \geqslant 1.96 \mathrm{M}_{\odot}$, which corresponds to the lower-limit estimate of the most massive neutron star known, the millisecond pulsar J0740+6620, at $95.4 \%$ confidence level [57]. In total, this yields the following 46 equations of state: ALF2, APR3, APR4, BCPM BSP, BSR2, BSR2Y, BSk20, BSk21, BSk22, BSk23, BSk24, BSk25, BSk26, DD2, DD2Y, DDHd, DDME2, DDME2Y, ENG, FSUGarnet, G3, GNH3, IOPB, K255, KDE0v1, MPA1, Model1, Rs, SINPA, SK272, SKOp, SKa, SKb, SLY2, SLY230a, SLY4, SLY9, SLy, SkI2, SkI3, SkI4, SkI6, SkMP, WFF1 and WFF2 [21, 22].

Our numerical code to calculate neutron stars (in general relativity and dynamical Chern-Simons gravity) uses the piecewise-polytrope approximation to model these equations of state [21]. In this approximation, the relationship between pressure $\left(p_{i}\right)$ and baryonic mass density $\left(\rho_{i}\right)$ is given by $p_{i}=K_{i} \rho^{\Gamma_{i}}$, where $K_{i}$ is the polytropic index and $\Gamma_{i}$ the adiabatic index, on a sequence of baryonic mass density intervals $i$. For the high-baryonic mass densities describing the neutron star core $\left(\rho_{\text {core }} \gtrsim 1.7 \times 10^{14} \mathrm{~g} / \mathrm{cm}^{3}\right)$, the exact value depends on the equation of state) we used a three-segment approximant, fitted to the equation of states listed above. For lowbaryonic mass densities $\left(\rho<\rho_{\text {core }}\right)$, matter is described using a four-segment approximant fitted against the SLy equation of state [21, 22].

One could argue [58] that a more rigorous approach to do inference on the nuclear equation of state from neutron star observations is to not use hard cuts as we do here. However, since our approach makes use of equation-of-state independent relations, changes to our equation of state catalog imply only small changes to the numerical values of the fitting coefficients. The hard cuts then have a negligible impact on our inferences, since the uncertainty on our quoted results (e.g., in Table I) are dominated by the uncertainties on the observations, except for the eccentricity $e_{\star}$.

Neutron-star catalog. - For each equation of state, we calculate a sequence of 50 neutron stars, spanning central baryonic mass densities in the range $\rho_{\mathrm{c}} \in[1.5,8.0] \rho_{\text {nuc }}$ (where $\rho_{\text {nuc }}=2.8 \times 10^{14} \mathrm{~g} / \mathrm{cm}^{3}$ is the nuclear saturation density) in general relativity and in dynamical Chern-Simons gravity. To obtain neutron star solutions in dynamical Chern-Simons gravity we use the same perturbative expansion in small-spin $(\varepsilon \ll 1)$ as used in general relativity, outlined in the main text, but combined with a small-coupling $(\zeta \ll 1)$ approximation as required for the well-posedness of the theory [59]. We then retained only those with (i) $M \leqslant M_{\max }$ (i.e. linearly stable against radial perturbations) and (ii) with $\mathscr{C} \in[0.125,0.3125]$ (i.e. within the prior on $\mathscr{C}$ used in the parameter estimation of PSR J0030+0451 by the Illinois-Maryland MCMC samples [26]). In the case of dynamical Chern-Simons gravity only we further imposed (iii) that all stars have $\zeta \leqslant 0.2$ (the small-coupling approximation). Although we used a $\mathscr{C}$ interval in (ii) based on [26] we note that $97 \%$ of the MCMC samples of the analysis by Riley et al. [7] do fall within this range. Therefore, we use the same $\mathscr{C}$ relations to make inferences with both MCMC samples, instead of deriving two sets of $\mathscr{C}$ relations based on the two NICER analysis [7, 8].

The $\mathscr{C}$ relations.- Relations among some stellar observables (e.g., moment of inertia, Love number or quadrupole moment) that do not depend sensitively on the equation-ofstate are called quasi-universal relations [60]. These relations are of the form

$$
y=\sum_{i=0}^{n} a_{i} x^{i}
$$

where $y$ and $x$ are particular stellar observables for which the relation exists, and $a_{i}$ are constant coefficients. In this work we use the " $\mathscr{C}$ relations" [12-15], shown in Fig. 3, with $(y, x)=$ $\left\{\left(\log _{10} \bar{I}_{\mathrm{GR}}, \mathscr{C}^{-1}\right),\left(\mathscr{C}, \log _{10} \bar{\lambda}_{\mathrm{GR}}\right),\left(\log _{10} \bar{Q}_{\mathrm{GR}}, \mathscr{C}\right),(e, \mathscr{C})\right\}$. The values of the coefficients $a_{i}$, the number of coefficients $n$, and the mean $\left\langle\epsilon^{y}\right\rangle$ and maximum relative error $\epsilon_{\max }^{y}=\max \left|1-y / y_{\text {fit }}\right|$ of the resulting fits are presented in Table II. In comparison to the I-Love relation, the $\mathscr{C}$ relations are slightly "less" quasi-universal (10\% versus $1 \%$ maximum relative error). However, their typical mean relative error at the $1 \%$ percent level justifies their use for parameter inference. We also verified explicitly that the $\mathscr{C}$ relations are weakly dependent on our equation of state catalog. This was verified by comparing the resulting fits (and associated errors) obtained using the initial full set of 85 equations and its subset of 46 .

Parameter inference: PSR J0030+0451.- To calculate the posterior distributions for $y=\{\bar{I}, \bar{\lambda}, \bar{Q}, e\}$, we follow the procedure used in [22]. In this scheme, the maximum relative error $\epsilon_{\max }^{y}$ (cf. the last column of Table II) is used to define the half-width of the $90 \%$ credible interval of a Gaussian distribution centered at each fitted value $\left(y_{\mathrm{fit}}\right)$,

$$
P(y \mid \mathscr{C})=\left(2 \pi \sigma_{y}^{2}\right)^{-1 / 2} \exp \left[-\left(y-y_{\mathrm{fit}}\right)^{2} /\left(2 \sigma_{y}^{2}\right)\right],
$$

where $\sigma_{y}=\epsilon_{\max }^{y} y_{\text {fit }} / 1.645$.

The posterior distribution for each dimensionless quantity, $P(y \mid$ NICER $)$, is then calculated using the corresponding $\mathscr{C}$ relation and the posterior probability distribution of the compactness, $P(\mathscr{C} \mid$ NICER) (see Fig. 4), obtained directly using NICER's MCMC samples [26, 27], and then marginalizing over $\mathscr{C}$ :

$$
P(y \mid \mathrm{NICER})=\int P(y \mid \mathscr{C}) P(\mathscr{C} \mid \mathrm{NICER}) \mathrm{d} \mathscr{C} .
$$

What dominates the uncertainty on our inferred values for the neutron star parameters? Is it the uncertainties associated 

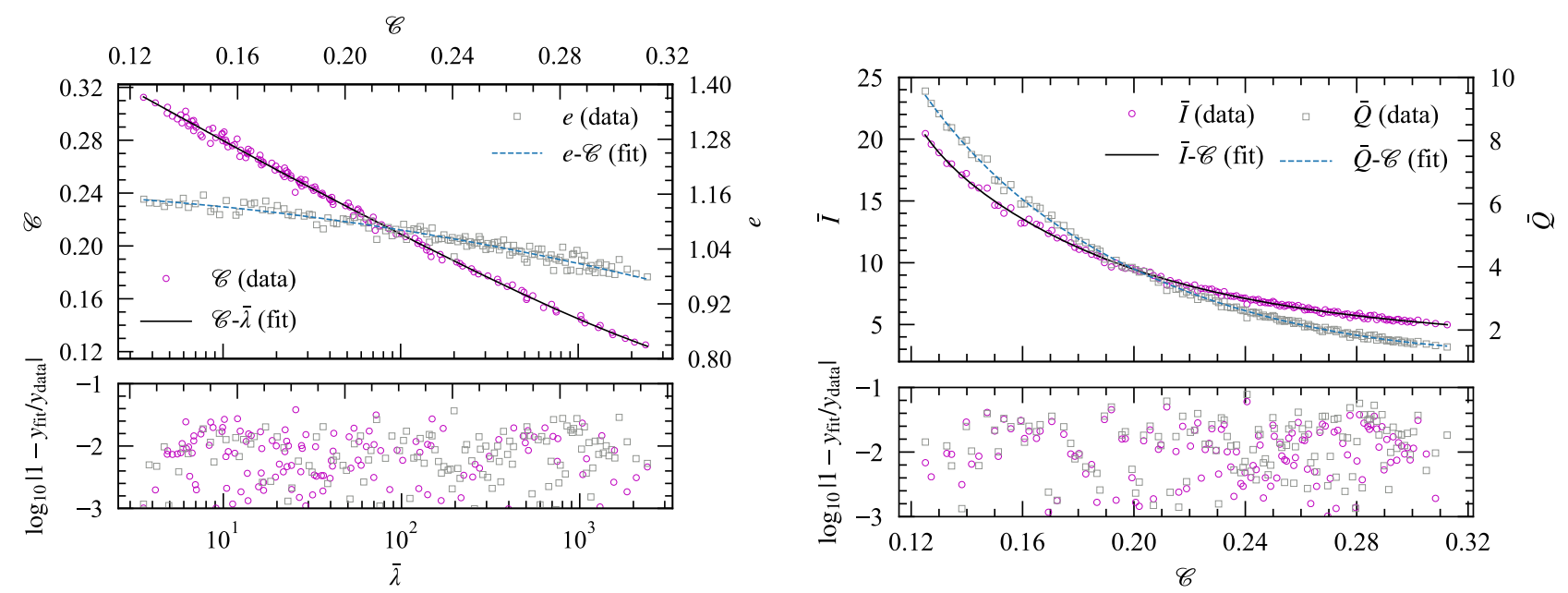

FIG. 3. The $\mathscr{C}$ relations. The top-left panel shows the relation between tidal Love number $\bar{\lambda}$ and compactness $\mathscr{C}$ (bottom and left axes) and between eccentricity $e$ and compactness $\mathscr{C}$ (top and right axes). The top-right panel shows the relation between the moment of inertia $\bar{I}$ (left axes), rotational quadrupole moment $\bar{Q}$ (right axes) and compactness $\mathscr{C}$. The lower panels show the relative errors $\left(\epsilon=\left|1-y_{\text {fit }} / y_{\text {data }}\right|\right)$ between the data and the fits. The largest mean relative error among the four $\mathscr{C}$ relations is $2 \%$ (corresponding to the Q- $\mathscr{C}$ relation), which shows the equation-of-state insensitivity of the relations.

\begin{tabular}{|c|c|c|c|c|c|c|c|c|c|}
\hline$y$ & $x$ & $n$ & $a_{0}$ & $a_{1}$ & $a_{2}$ & $a_{3}$ & $a_{4}$ & $\left\langle\epsilon^{y}\right\rangle$ & $\epsilon_{\max }^{y}$ \\
\hline $\log _{10} \bar{I}_{\mathrm{GR}}$ & $\mathscr{C}^{-1}$ & 4 & $5.5531 \times 10^{-1}$ & $-1.7705 \times 10^{-1}$ & $1.1105 \times 10^{-1}$ & $-1.5137 \times 10^{-2}$ & $6.9401 \times 10^{-4}$ & $1.52 \times 10^{-2}$ & $6.7 \times 10^{-2}$ \\
\hline $\mathscr{C}$ & $\log _{10} \bar{\lambda}_{\mathrm{GR}}$ & 4 & $3.5818 \times 10^{-1}$ & $-8.8149 \times 10^{-2}$ & $1.3120 \times 10^{-2}$ & $-4.5810 \times 10^{-3}$ & $6.9924 \times 10^{-4}$ & $9.63 \times 10^{-3}$ & $4.2 \times 10^{-2}$ \\
\hline $\log _{10} \bar{Q}_{\mathrm{GR}}$ & $\mathscr{C}$ & 4 & 2.1088 & $-1.4669 \times 10^{1}$ & $6.6952 \times 10^{1}$ & $-2.1050 \times 10^{2}$ & $2.6576 \times 10^{2}$ & $1.91 \times 10^{-2}$ & $8.6 \times 10^{-2}$ \\
\hline$e$ & $\mathscr{C}$ & 2 & 1.1624 & $2.0527 \times 10^{-1}$ & -2.5870 & - & - & $9.86 \times 10^{-3}$ & $4.1 \times 10^{-2}$ \\
\hline
\end{tabular}

TABLE II. Numerical coefficients for the fits to the $\mathscr{C}$ relations. These fits are tailored to the Illinois-Maryland MCMC data samples [26] and, therefore, are only valid within the compactness range $\mathscr{C} \in[0.125,0.3125]$.

with the $\mathscr{C}$-measurement we are using, or those introduced by the approximate universality of the $\mathscr{C}$ relations? To answer this question, in Figure 5 we show the resulting posterior distributions by taking into account the approximate universality of the $\mathscr{C}$ relations (solid lines) and compare against the posterior distributions on the same parameters if we do not (dashed lines). In the latter case, the posteriors are obtained by directly applying the $M-R_{\mathrm{e}}$ samples [26] in the $\mathscr{C}$ relations. The effect of including the systematic error induced by the variability of the $\mathscr{C}$ relations is to broaden the posterior, a small effect for $\bar{I}, \bar{\lambda}$ and $\bar{Q}$, but not for the eccentricity $e$. The reason is the following: the mean relative error for the e- $\mathscr{C}$ fit is approximately $4 \%$, which is larger than the variability of $e$ in the $1 \sigma$ interval of its distribution. This leads to the considerable broadening shown in the bottom-right panel of Fig. 5.

The posteriors for the dimensionful version of these quantities are obtained of through a change of variables, and using the posteriors on $M$ and $R_{\mathrm{e}}$. For instance, for the moment of inertia $I$,

$$
\begin{aligned}
P(I \mid \mathrm{NICER})= & \left(c^{2} / G\right)^{2} \int P\left(c^{4} I /\left(G^{2} M^{3}\right) \mid \mathrm{NICER}\right) \\
& \times P(M \mid \mathrm{NICER}) M^{-3} \mathrm{~d} M .
\end{aligned}
$$

The posterior distributions for the dimensionful variables are similar in shape to their nondimensionalized versions.

Parameter inference: fixed mass. - To calculate the posterior distribution of a quantity $y$ for a neutron star with known mass $M_{0}$ using the $\mathscr{C}$ relations, we first need to contruct a posterior $P\left(\mathscr{C}_{M_{0}} \mid\right.$ NICER $)$ given $P(\mathscr{C} \mid$ NICER $)$. To do so, we write

$$
P\left(\mathscr{C}_{M_{0}} \mid \mathrm{NICER}\right)=P\left(\left[G M /\left(R_{\mathrm{e}} c^{2}\right)\right]_{M=M_{0}} \mid \mathrm{NICER}\right),
$$

where the right-hand side is obtained by using the MCMC samples [26], doing the substitution $\left\{M, R_{\mathrm{e}}\right\} \mapsto\left\{M_{0}, R_{\mathrm{e}}\right\}$ at each sample and then computing $\mathscr{C}_{M_{0}}$. This procedure gives an approximation to the distribution of compactness for a system with known mass $M_{0}$, which we here take to be identical to PSR J0030+0451. Of course, the posterior $\mathscr{C}_{M_{0}}$ obtained in this way would fail dramatically if the difference between $M_{0}$ and PSR J0030+0451's mass, $M_{\star}=1.44_{-0.14}^{+0.15} \mathrm{M}_{\odot}[8]$, is large. However, this is not case for a canonical neutron star with mass $1.4 \mathrm{M}_{\odot}$ and PSR J0737-3039A's, which has a mass of $1.3381 \mathrm{M}_{\odot}$ masses, both of which agree with $M_{\star}$ within $1 \sigma$.

We can justify this more quantitatively as follows. First, for each equation of state we compute the compactness for each mass $m_{1}$ and $m_{2}$. For our case, this would produce 46 points in the $\mathscr{C}_{1}$ and $\mathscr{C}_{2}$ plane, corresponding to the 46 equations of state we use. Then, we fit a line to these points and calculate the maximum residual from the best-fit line. This 


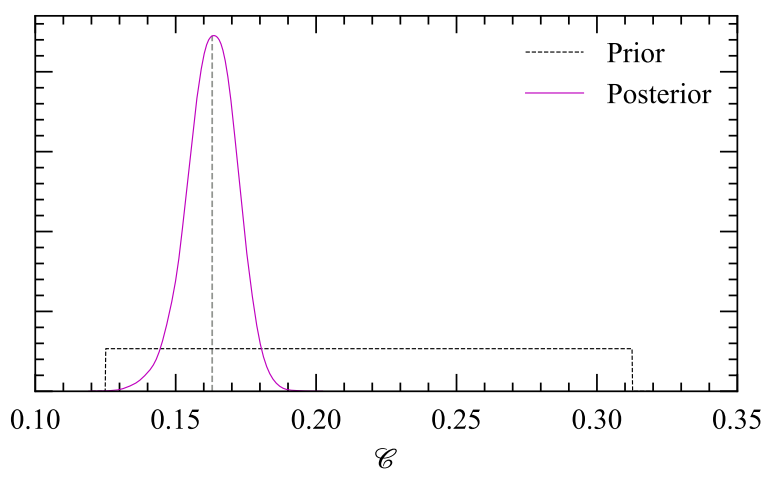

FIG. 4. Prior and posterior probability distributions on the compactness of PSR J0030+0451 $\left(\mathscr{C}_{\star}\right)$. The Illinois-Maryland analysis $[8,26]$ assumed a flat prior probability distribution for $\mathscr{C}_{\star}$ with bounds 0.125 and 0.3125 (dashed curve). The posterior probability distribution (solid line) has a median value of $\mathscr{C}_{\star}=0.163$ [8]. The posterior is approximately Gaussian and very different from the flat (uninformative) prior used by NICER, showing that the NICER observation was indeed informative.

procedure can be done for any combinations of $m_{1}$ and $m_{2}$. We plot the maximum residual for the estimated compactness for different $m_{1}$ and $m_{2}$ in Fig. 6. The mass ratio between PSR J0030+0451 and both PSR J0737-3039A and a $1.4 \mathrm{M}_{\odot}$ star is approximately 0.9 for which the maximum residual is about $1 \%$.

Knowing $P\left(\mathscr{C}_{M_{0}} \mid\right.$ NICER $)$, we can use it in Eq. (6) and marginalize over $\mathscr{C}_{M_{0}}$ to obtain the posterior distribution of a quantity $y$ at mass $M_{0}$. Our results for $\mathscr{C}, R_{\mathrm{e}}$ and $y=\{\bar{I}, \bar{\lambda}$, $\bar{Q}, e\}$ for a canonical neutron star are summarized in Table III.

I-Love relation in dynamical Chern-Simon gravity. - Let us describe further how to map the theory-agnostic deformation of the I-Love relations in Eq. (2) to dynamical Chern-Simons gravity. The procedure outlined here can be applied to other theories as well.

The dimensionless moment of inertia of a constant density star in dynamical Chern-Simons gravity is $\bar{I}=\bar{I}_{\mathrm{GR}}+\bar{I}_{\mathrm{CSN} N}$, where $\bar{I}_{\mathrm{CS}, \mathrm{N}}=C_{\overline{\bar{L}} \bar{\lambda}}^{\mathrm{CS} \mathrm{N}} \alpha^{2} M / R^{5}$ and $C_{\bar{I} \bar{\lambda}}^{\mathrm{CS}, \mathrm{N}}=1024 \pi / 75$ [61] to leadingorder in $\alpha$ and leading-order in $\mathscr{C}$. The I-Love relations in dynamical Chern-Simons gravity are insensitive to the equation of state when $\bar{I}_{\mathrm{CS}}$ is normalized via $\bar{\xi} \equiv 16 \pi \alpha^{2} / M^{4}$ [62]. Therefore, using that $\bar{\lambda}_{\mathrm{N}}=(1 / 2) \mathscr{C}^{-5}$ in Newtonian gravity for a constant density star, we then have that $\bar{I}_{\mathrm{CS}, \mathrm{N}}=$ $(16 \pi)^{-1} C_{\bar{I} \bar{\lambda}}^{\mathrm{CSN}} \bar{\xi} \mathscr{C}^{5}=\left[(32 \pi)^{-1} C_{\bar{I} \bar{\lambda}}^{\mathrm{CSN}} \bar{\xi}\right] \bar{\lambda}^{-1}$. Although this relation was derived assuming a constant density star, it holds for any equation of state, with variability at the sub-percent level [62]. Comparison of this result to Eq. (2) reveals that the mapping between our proposed parameterization and dynamical Chern-Simons gravity is $\beta_{\mathrm{CS}, \mathrm{N}}=(32 / 75) \bar{\xi}$ and $b_{\mathrm{CS}, \mathrm{N}}=5$.

How well does this "Newtonian" approximation capture the fully relativistic I-Love relation in dynamical Chern-Simons gravity? Figure 7 shows that the Newtonian approximation derived above is excellent for low-mass neutron stars. The scaling with $\bar{\lambda}^{-1}$, however, fails for more massive stars, when $M \gtrsim 1 \mathrm{M}_{\odot}$, because relativistic corrections become impor-
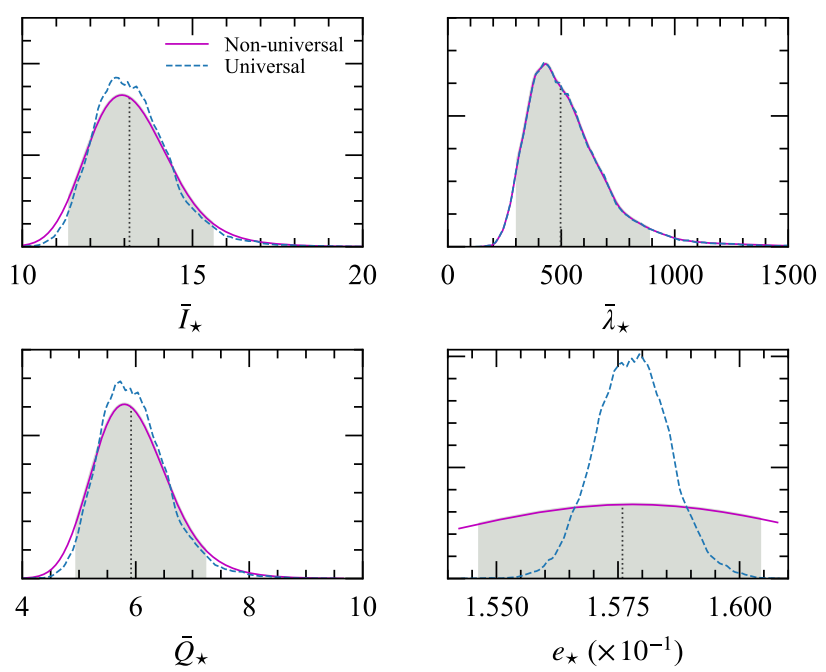

FIG. 5. Posterior distributions on the dimensionless quantities $\{\bar{I}$, $\bar{\lambda}, \bar{Q}, e\}$ of PSR J0030+0451. We show both the distributions obtained by taking into account the systematic error introduced by the quasiuniversality of the $\mathscr{C}$ relations (solid lines) and that assuming the universality of the relations (dashed lines). The vertical lines and the shaded bands represent the mean and $90 \%$ posterior credible intervals, respectively. The posteriors show that for $\bar{I}, \bar{\lambda}$ and $\bar{Q}$, the uncertainties associated with our inferences are dominated by the uncertainty on the measured value $\mathscr{C}_{\star}$. This is not the case for $e$, because the systematic error associated with the e- $\mathscr{C}$ fit is larger than that associated with the inference of $e$, assuming complete equationof-state independence on the e- $\mathscr{C}$ relation.

tant. In spite of this, the dynamical Chern-Simons correction to the relativistic I-Love data, for values of $\bar{\lambda}$ corresponding to stars with compactness $\mathscr{C}$ within NICER's priors, can be well-approximated by $\bar{I}_{\mathrm{CS}}=\beta_{\mathrm{CS}} \bar{\lambda}^{-b_{\mathrm{CS}} / 5}$, with

$$
\beta_{\mathrm{CS}}=6.15 \times 10^{-2} \bar{\xi}, \quad b_{\mathrm{CS}}=4 .
$$

This functional form is quite close to the Newtonian result (in fact, just a factor of $\mathscr{C}$ higher than the Newtonian result because $\mathscr{C} \propto \bar{\lambda}^{-1 / 5}$ ), which suggests the rule of thumb $b=b^{\mathrm{N}}-$ 1 , where $b^{N}$ is the result of the I-Love calculation to Newtonian order in modified gravity.

By virtue of the parity properties of the field equations in dynamical Chern-Simons gravity, the electric-type $\ell=2$ tidal Love number in this theory is identical to that in general relativity for nonrotating neutron stars.

Using the results from Riley et al. [7, 27], we obtain the same bound on dCS found in the main text, namely $\sqrt{\alpha} \leqslant$ $8.5 \mathrm{~km}$. We also find that $\zeta \leqslant 0.26$, only $4 \%$ above our threshold for the small-coupling approximation, however still $\ll 1$.

Constraints on the parametrized I-Love parameters- In Table IV we summarize the numerical values for the contrainst of the parametrized I-Love parameters $b$ and $\beta$.

The parametrized I-Love test and why can neutron stars constrain dynamical Chern-Simons gravity- Here we explain the rationale behind the parametrized I-Love test of gravity and why it can be applied to dynamical Chern-Simons gravity. Perhaps the easiest way to understand the parametrized 


\begin{tabular}{lcccc}
\hline \hline Parameter & Source / Universal relation & Median & $-1.65 \sigma$ & $+1.65 \sigma$ \\
\hline $\mathscr{C}_{1.4}\left(10^{-1}\right)$ & MCMC chain / & 1.59 & 1.37 & 1.84 \\
$R_{1.4}(10)[\mathrm{km}]$ & MCMC chain / & 1.30 & 1.12 & 1.51 \\
\hline $\bar{I}_{1.4}(10)$ & MCMC chain / I- $\mathscr{C}$ & 1.46 & 1.12 & 1.91 \\
$\bar{\lambda}_{1.4}\left(10^{2}\right)$ & MCMC chain / Love- $\mathscr{C}$ & 5.80 & 2.33 & 13.7 \\
$\bar{Q}_{1.4}$ & MCMC chain / Q- $\mathscr{C}$ & 6.12 & 4.52 & 8.12 \\
$e_{1.4,0}$ & MCMC chain / e- $\mathscr{C}$ & 1.12 & 1.09 & 1.15 \\
\hline \hline
\end{tabular}

TABLE III. Properties of $M=1.4 \mathrm{M}_{\odot}$ neutron stars, inferred from PSR J0030+0451 at $90 \%$ credibility. The eccentricity $e_{1.4,0}$ is evaluated at the characteristic mass-shedding frequency $f_{0}$. To rescaled it to the eccentricty $e_{1.4}$ of a star spinning with frequency $f$ one has to multiply by $\varepsilon=f / f_{0}=1532.7 \mathrm{~Hz}$.

\begin{tabular}{c|cccccccc}
\hline \hline$b$ & -2 & -1 & 0 & 1 & 2 & 3 & 4 & 5 \\
\hline$\beta_{\text {crit }}$ (Miller et al.) & 1.97 & 4.63 & 10.8 & 25.7 & 74.5 & $2.49 \times 10^{2}$ & $8.84 \times 10^{2}$ & $3.15 \times 10^{3}$ \\
$\beta_{\text {crit }}$ (Riley et al.) & 1.99 & 4.67 & 11.0 & 26.0 & 75.5 & $2.53 \times 10^{2}$ & $9.00 \times 10^{2}$ & $3.21 \times 10^{3}$ \\
\hline \hline
\end{tabular}

TABLE IV. Constraints on the parametrized I-Love relation. For a given exponent $b$, parametrized I-Love curves with $\beta \geqslant \beta_{\text {crit }}$ do not pass through the $90 \%$ credible intervals on the inferred values of $\bar{I}_{1.4}$ and $\bar{\lambda}_{1.4}$ as shown in Fig. 2 . The constraints obtained using the results of both Miller et al. [8] and Riley et al. [7] are similar.

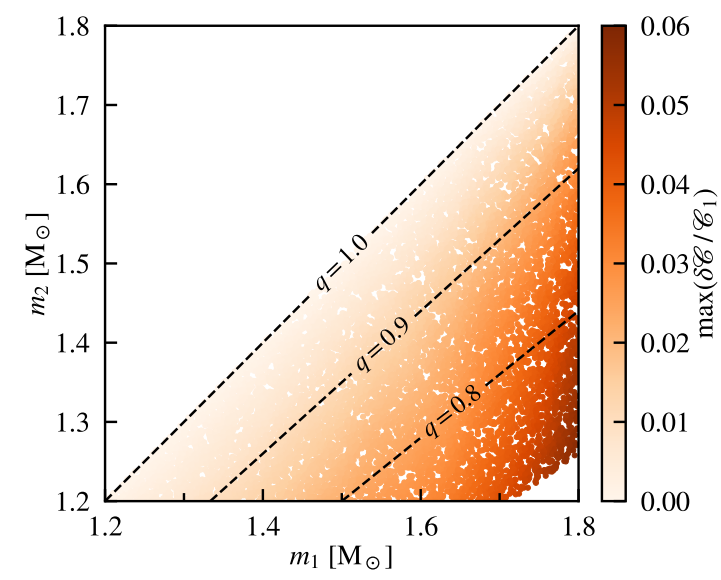

FIG. 6. Maximum residual when predicting the compactness $\mathscr{C}_{2}$ of a NS with $m_{2}$ given $m_{1}$ and $\mathscr{C}_{1}$. The dashed contours correspond to lines of constant mass ratio $q=m_{2} / m_{1}$. The mass ratio between PSR J0030+0451 and both a PSR J0737-3039A and a $1.4 \mathrm{M}_{\odot}$ star is about 0.9 . Overall, the largest residual is about 0.06 in the mass range shown.

I-Love test is to present it alongside with the parametrized post-Keplerian formalism (ppK) used to test general relativity with binary pulsars [63]. Both are very similar in spirit. In the ppK formalism, a timing model that assumes Keplerian orbits augmented with certain theory-agnostic post-Keplerian parameters $\boldsymbol{\theta}$ is fit to timing data. This provides marginalized posterior distributions for all parameters in the timing model, including both the orbital ones (such as the orbital period $P_{b}$ and eccentricity $e$ ), as well as the post-Keplerian ones (such as the Shapiro time delay $\gamma$ and the secular advance of periastron $\dot{\omega})$. To do a theory-agnostic, general relativity test with binary-pulsar data, one then uses expressions that assume general relativity is correct for how the post-Keplerian parameters

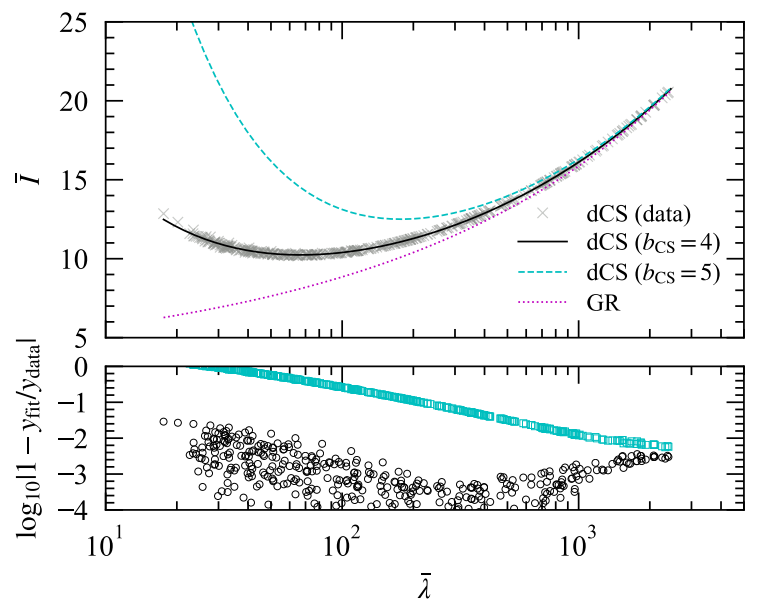

FIG. 7. I-Love relation in dynamical Chern-Simons gravity. In the top-panel we show the numerical data (crosses) obtained for neutron stars in dynamical Chern-Simons, the I-Love relation in general relativity (dotted line) and fits to it using the Newtonian result obtained in the main text (dashed line) and the post-Minkowskian corrected result (solid line). In the bottom-panel we show the relative errors between both fits and the data, explicitly showing that the Newtonian fit cannot be used for an I-Love test. In this figure we fixed $\bar{\xi}=10^{3}$ and only show stars with $\zeta \leqslant 0.25$.

depend on the component masses $m_{A}$ and $m_{B}$. These expressions then define curves in $m_{A}-m_{B}$ space (one curve per postKeplerian parameter), and under the assumption that general relativity is correct, then all curves must interest at the same point in $m_{A}-m_{B}$ space (see e.g. [29]). In practice, the curves have a width since the measurements are not perfect, so the intersections of the curves define a two-dimensional region in $m_{A}-m_{B}$ space.

Let us provide a concrete example. Assuming general rel- 
ativity, the post-Keplerian parameters $\dot{\omega}, \gamma$ and $r$ (the range of the Shapiro time delay) are $\dot{\omega}=3 n\left(1-e^{2}\right)^{-1}\left(G M n / c^{3}\right)^{2 / 3}$, $\gamma=(e / n) X_{B}\left(G M n / c^{3}\right)^{2 / 3}\left(1+X_{B}\right)$ and $r=G m_{B} / c^{3}$, where $X_{A, B}=m_{A, B} / M$ is the mass ratio, with the total mass $M=$ $m_{A}+m_{B}$ and component masses $m_{A}$ and $m_{B}$, while $e$ is the orbital eccentricity and $n=2 \pi / P_{b}$ is the orbital frequency with $P_{b}$ the orbital period. Since the orbital period and the eccentricity are independent parameters in the timing model that are, therefore, included in the Bayesian parameter estimation, the above expressions represent 3 curves in $m_{A}-m_{B}$ space. The curves have a width that is dominated by the uncertainty in the measurements of $\gamma, \dot{\omega}$ and $r$, so if the assumption that general relativity is correct, these three finite-width curves must intersect and define a two-dimensional region in $m_{A}-m_{B}$ space.

Our theory-agnostic test is very similar to this one. In our approach, the quantities that are independently measured are the moment of inertia $I$ and the Love number $\lambda$, which require two independent observations (NICER and LIGO/Virgo ones, instead of a single binary pulsar observation). These two measurements define a two-dimensional region in $I-\lambda$ space, whose finite size is associated with the uncertainty in these observations. The moment of inertia and the Love number also satisfy a relation, the I-Love relation, which is (approximately) independent of the equation of state, but dependent on the theory of gravity. Therefore, the relation constructed assuming general relativity must cross the two-dimensional box in $I-\lambda$ space defined by the two observations if general relativity is correct. This does indeed happen as we showed for the first time in Fig. 2 of the main text.

How do these tests work to constrain a specific modifiedgravity theory? In the binary pulsar case, the timing model used to analyze the data does not change: it is still the Keplerian model enhanced with post-Keplerian parameters $\boldsymbol{\theta}$. What changes is the relation between these post-Keplerian parameters and the component masses, a relation that will now also typically include additional modified theory parameters. For example, in scalar-tensor theories [64] that support spontaneous scalarization [65], this relation now also depends on the spontaneous scalarization parameter $\beta$ (see e.g. [66, 67]). Therefore, the observation of three post-Keplerian parameters allows one to infer a constraint on $\beta$, since if this parameter were large (and negative) enough to generate a strong deviation from general relativity, then the three post-Keplerian parameter curves would not intersect in the $m_{1}-m_{2}$ space.

Our constraint on specific modified-gravity theories operates in a similar way, and just for the sake of concreteness, let us consider dynamical Chern-Simons gravity as a simple example. We will assume that the model used to fit the data (i.e. the lightcurve model used by NICER and the gravitational waveform model used by LIGO/Virgo) is that assumed in general relativity. This leads to a two-dimensional region in $I-\lambda$ space, just as in the theory-agnostic case. What changes now is that the $I-\lambda$ relation is not just a function of the Love number, but it also depends on the coupling parameter $\alpha$. The larger this parameter is, the more the $I-\lambda$ curve will deviate from the observed two-dimensional region in $I-\lambda$ space. One can then determine how small $\alpha$ must be in order to be con- sistent with the $I$ and $\lambda$ observations, and this is the constraint quoted in the main text. We summarize this discussion in Table $\mathrm{V}$.

Is our assumption that the model used to fit the NICER and LIGO/Virgo data is not modified from general relativity a good assumption to make in this test? Let us first continue considering dynamical Chern-Simons gravity. In this theory it has been shown that neutron stars do not have (monopole) scalar hair [68]. Therefore one cannot use the gravitational waves emitted during the inspiral of binary neutron stars to test dynamical Chern-Simons gravity due to the absence of dipole scalar radiation emission. Moreover, the light-curve model used by NICER considers the emission of X-rays from an oblate star surface and with photons propagating in a Schwarzschild spacetime (the so-called "Oblate+Schwarscshild model" [16]). On the one hand, nonrotating stars in dynamical Chern-Simons gravity are identical to those of general relativity, and therefore, their exterior spacetime is also described by the Schwarzschild metric [69]. On the other hand, the stellar oblateness (due to rotation) could in principle be used to distinguish this theory gravity from general relativity. However modifications to the oblateness due to changing the theory of gravity are degenerate with assuming general relativity but changing the underlying equation of state. Together, these points justify our assumption that the model used to fit the NICER and LIGO/Virgo data is not modified from general relativity in dynamical Chern-Simons gravity.

What about other theories? In the case of scalar-tensor theories, which support spontaneous scalarization (exemplified above), neutron stars have (monopole) scalar hair and therefore, in general, emit dipolar scalar radiation when found in binaries; and they also have an exterior spacetime that differs from the Schwarzschild metric. In this case, a direct confrontation of the models in this theory against observational data could yield a constraint on $\beta$ using observations by NICER (see [70-72] and particularly [73] for initial work in this direction). Such constraints are likely to be stronger than the ones obtained using our parametrized I-Love test. This is specially true for theories whose lightcurve model and gravitational waveforms are considerably different from general relativity. But none of this invalidates our I-Love test. At the very least, our I-Love test would provide complementary constraints to other more direct tests. More importantly, the I-Love test is a new tool to constrain theories of gravity which would remain unconstrained by NICER and gravitational wave observations alone, as we have shown for dynamical Chern-Simons gravity as an example. This fact highlights the importance of using multimessenger observations to constrain deviations from general relativity. Our parametrized ILove test is the first one in this direction.

Let us do a back-of-the-envelope estimate to explain why neutron star observations have such a constraining power in dynamical Chern-Simons gravity. Assume that $\zeta$ has been constrained to some value, say $\zeta \leqslant 0.25$, by some set of observations that involve also measurements of the mass and equatorial radius of a neutron star. A posterior distribution for $\alpha^{1 / 2}$ can then be obtained by translating the MCMC samples for $M$ 


\begin{tabular}{l|c|c}
\hline \hline & $\mathrm{ppK}$ & $\mathrm{pIL}$ \\
\hline Astrophysical system & binary pulsar & isolated NS and a NS binary \\
\hline Instrument & radio telescopes & NICER and LIGO/Virgo \\
\hline Model & theory-agnostic timing formula & GR light-curve and GW waveform \\
\hline Parameters & post-Keplerian $\boldsymbol{\theta}\left(m_{1}, m_{2}, \mathscr{P}\right)$ & $I=I(\lambda, \mathscr{P})$ \\
\hline $\begin{array}{l}\text { \# of parameter } \\
\text { measurements for test }\end{array}$ & $\begin{array}{c}\text { 3 (two for } m_{1}, m_{2}, \\
\text { third for test) }\end{array}$ \\
\hline \hline
\end{tabular}

TABLE V. Comparison between the parametrized post-Keplerian (ppK) and parametrized I-Love (pIL) relation tests of gravity. Here $\mathscr{P}$ collectively represents the parameters that control the deviations from general relativity. They all vanish for general relativity.

and $R_{\mathrm{e}}$ into samples of $\alpha^{1 / 2}=[\zeta /(16 \pi)]^{1 / 4} \mathscr{R}$. Using for concreteness the MCMC samples obtained by NICER, one would obtain $\alpha^{1 / 2} \leqslant 8.57_{-1.13}^{+0.93} \mathrm{~km}$ (at $90 \%$ credibility), which is very close to the real constraint derived in the main text. The backof-the-envelope estimate presented above is of course not an actual constraint since NICER alone cannot bound $\zeta$ due to degeneracies with the equation of state. But this estimate does tell us that such a test would be dominated by $\mathscr{R}$, because an improvement on the bound on $\zeta$ by a factor of $k$ would only improve the constraint on $\alpha^{1 / 2}$ by $k^{1 / 4}$. 